\section{Tuning holes in photonic-crystal nanocavities}

Arising from: Y. Akahane, T. Asano, B.-S. Song \& S. Noda Nature 425, 944-947 (2003)

0 ne challenge in photonics is strongly to confine light in small volumes in order to increase light-matter interaction. Akahane et al. ${ }^{1}$ propose a new concept for increasing the lifetime of this interaction, based on tailoring of the Fourier spectrum of cavity modes, which they believe is demonstrated by the surprising enhancement (roughly tenfold) of the quality factor $Q$ of the cavity as a result of fine-tuning the mirror-hole geometry in a photonic-crystal nanocavity. Here we question the validity of their concept and argue that the improvement in $Q$ is due to an increase in the impedance wave matching at the cavity edges and to a slow-wave effect. This alternative interpretation opens the way to new cavity designs.

Like Akahane et al. ${ }^{1}$, we first consider two cavities formed in a silicon slab with air claddings (Fig. 1a). The light is confined in the $x$-direction by perfect-metal mirrors (left cavity) or by Bragg mirrors (right cavity). For the cavity parameters given in the figure legend and for $y$-polarization, the resonant wavelength is $\lambda=1.5 \mu \mathrm{m}$. Using the Fourier modal method $^{2}$, we calculate the cavity mode profiles at the centre plane of the slab (Fig. 1b) and their Fourier spectra (Fig. 1c).

These curves closely resemble those in Fig. 2 of Akahane et al., which would suggest ${ }^{1}$ that the metallic cavity, with large Fourier components in the leaky region, should show a smaller $Q$ factor than the dielectric one.
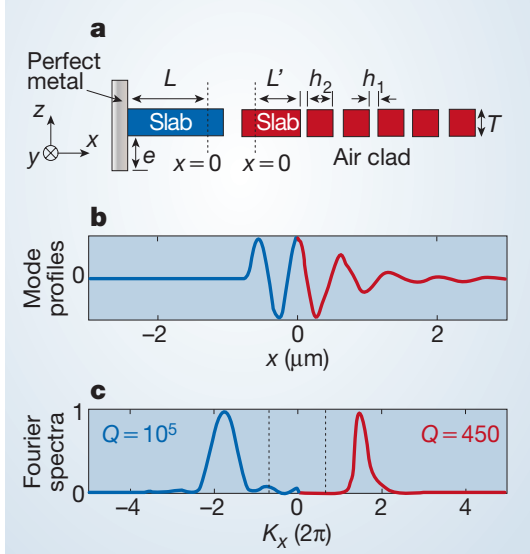

Figure 1 Counterexample for the Fourier space method. a, Cavity geometries. Parameters are: $T=0.2 \mu \mathrm{m}, e=0.5 \mu \mathrm{m}, 2 L^{\prime}=0.37$ $\mu \mathrm{m}, 2 L=1.38 \mu \mathrm{m}, h_{2}=0.25 \mu \mathrm{m}, h_{1}=0.1 \mu \mathrm{m}$; the silicon refractive index is 3.4. As both cavities are symmetric with respect to the $x=0$ plane, only half of each is represented. $\mathbf{b}$, Real part of the electric field profiles of the cavity mode at the centre plane of the slab. c, Modulus squared of the spatial Fourier spectra of the cavity modes. Vertical dashed lines delimitate the domain of leakage into the air clads, $\left|k_{x}\right|<2 \pi / \lambda$.
However, the metallic-cavity $Q$ is $10^{5}$, a value that is 200 times larger than that of the dielectric cavity. In addition, for a null metal height of $e$, the mode profile of the metallic cavity remains completely unchanged but its $Q$ is only 350 .

We infer that the mode profile inside the cavity conveys nothing about the mode lifetime. The Fourier spectrum method is valid for calculating mode profiles across a surface positioned above the cavity in the air $\mathrm{clad}^{3}$. For such $x$-invariant surfaces, the cavity-mode profile and its Fourier spectrum contain all the information on the far-field radiation losses.

But this is not true for a surface inside the cavity. The reason is that the parallel $k_{x}$ momentum of the cavity mode is not strictly matched at the slab-cladding interface because neither Snell's law nor Fresnel's law apply at a corrugated interface. Design rules that overlook this parallel-momentum mismatch ignore the essence of the longitudinal confinement and, in turn, the associated impedance-mismatch problem ${ }^{4}$ that determines radiation losses at the cavity edges.

To understand the physical reasons for the roughly tenfold enhancement in $Q$, we consider a Fabry-Perot model. We assume that the nanocavity mode is formed by the recirculation of the fundamental Bloch wave of the line-defect waveguide between the mirrors. The model, which relies on a three-dimensional computation ${ }^{2}$ for the Bloch-wave modal reflectivity $\left|r_{\mathrm{m}}\right| \exp (i \phi)$, reproduces the experimental trends well: a red-shift of $\lambda$ as the hole shift $d$ increases, and a peak value of $Q$ for $d \approx 0.17 a$, where $a$ is the lattice constant of the photonic crystal. For Fabry-Perot cavities ${ }^{5}$,

$$
Q=\frac{\pi}{1-\left|r_{\mathrm{m}}\right|^{2}}\left(\frac{2 L}{\lambda} n_{\mathrm{g}}-\frac{\lambda}{\pi} \frac{\delta \phi}{\delta \lambda}\right)
$$

where $L=3 a$ is the defect length. From equation (1), three effects explain the $Q$ improvement between $d=0$ and $0.17 a$ : an increase of $\left|r_{\mathrm{m}}\right|^{2}$ from $98.3 \%$ to $99.7 \%$, a doubling of the group-index $n_{\mathrm{g}}$ of the Bloch wave, and a doubling of the penetration depth $\delta \phi / \delta \lambda$ into the mirrors.

The first effect has been analysed previously for a one-dimensional slab ${ }^{6}$ and twodimensional air-bridge cavities ${ }^{7}$, and the second and third effects result from the highly dispersive nature ${ }^{8,9}$ of the Bloch wave in the spectral window. Our analysis highlights the importance of wave impedance matching in nanocavities and the key role of slow waves in further improvements. Christophe Sauvan, Philippe Lalanne, Jean-Paul Hugonin
Laboratoire Charles Fabry de l'Institut Optique, Centre National de la Recherche Scientifique et Université Paris Sud, BP 147,

91403 Orsay Cedex, France

e-mail:philippe.lalanne@iota.u-psud.fr

doi:10.1038/nature02602

1. Akahane, Y., Asano, T., Song, B.-S. \& Noda., S. Nature 425, 944-947 (2003).

2. Silberstein, E., Lalanne, P., Hugonin, J. P. \& Cao, Q. J. Opt. Soc Am. A 18, 2865-2875 (2001).

3. Vuckovic, J., Loncar, M., Mabuchi, H. \& Scherer, A. IEEE J. Quant. Electr. 38, 850-856 (2002).

4. Palamaru, M. \& Lalanne, P. Appl. Phys. Lett. 78, 1466-1468 (2001)

5. Coldren, L. A. \& Corzine, S. W. (eds) Diode Lasers and Photonic Integrated Circuits (Wiley, New York, 1995).

6. Lalanne, P. \& Hugonin, J.-P. IEEE J. Quant. Electr. 39, 1430-1438 (2003).

7. Lalanne, P., Mias, S. \& Hugonin, J. P. Opt. Exp. 12, 458-467 (2004).

8. Notomi, M. et al. Phys. Rev. Lett. 87, 253902 (2001).

9. Chutinan, A. \& Noda, S. Phys. Rev. B 62, 4488-4492 (2000).

Akahane et al. reply - In response to Sauvan et al. ${ }^{1}$, we perceive a flaw in their use of the Fourier analysis method and confirm that the increased $Q$ factor in a nanocavity with tuned air holes is based on our design concept ${ }^{2}$.

Referring to the cavity shown with a metal mirror in Fig. 1 of Sauvan et al., the slab cavity is surrounded by free space (or air clad) in the vertical direction when $e=0$, which corresponds to the case $e^{2}$ in our Fig. 2b, c. The lifetime of the cavity mode is determined by the amount of its Fourier components inside the 'leaky region' determined by air clad. When $e$ becomes non-zero, however, the situation changes drastically, because the outer space of the slab cavity changes from the air clad to a hollow cavity (or waveguide) that is surrounded by metals and the slab surface ${ }^{3}$. It means that the leaky region is no longer determined by the air clad but by the hollow cavity. Because the number of modes in the hollow cavity ${ }^{3}$ is much less than that of those in air clad, the leaky region is very much reduced. Thus, the leakage of light from the slab cavity to the outer space is decreased, and the $Q$ factor increases.

Sauvan et al. overlook this change in the leaky region that is associated with the change in the outer space, which leads to a serious misunderstanding. The method itself (to increase the $Q$ factor) is interesting, but in reality the metal mirror has considerable absorption and is hard to use for an optical regime. Thus, we concentrated on the Bragg reflector cavity type ${ }^{2}$.

When we investigate the mode lifetime of the Bragg reflector cavity surrounded by air clad, we can apply a normal Fourier analysis method by using the leaky region determined by the air clad. We described an important design concept ${ }^{2}$ in which the envelope of the in-plane mode profile in the cavity should be varied gently but localized spatially (gaussianlike, lorentzian-like or like the related function) to increase $Q$ while keeping a small 